\title{
The effect of Maras powder and smoking on the microRNA deregulation of oral mucosa
}

\section{Abstract}

Betül TAŞ ${ }^{1}$

Ali Osmay GÜRE²
Submitted: July 11, 2019 Modification: October 22, 2019 Accepted: October 23, 2019

Corresponding address: Betül Taş

Universite Bulvari, Osmangazi mah - Gaziantep Universitesi Dis Hekimligi Fakultesi - Ağiz Dis ve Cene Hastaliklari Cerrahisi, 27310 - Gaziantep - Turkey. Phone: 00905303460713 - Fax: 00903423610346 e-mail: betultas@hotmail.com - tasbetul@gmail.com
${ }^{1}$ Gaziantep University, Faculty of Dentistry, Department of Oral and Maxillofacial Surgery, Gaziantep, Turkey.

${ }^{2}$ Bilkent University, Department of Molecular Biology and Genetics, Ankara, Turkey.
Objective: This study aimed to investigate the effects of Maras powder (a type of smokeless tobacco obtained from Nicotiana rustica Linn and mixed with the ashes of wood, especially from oak, walnut or grapevine) on the microRNA (miRNA) deregulation of oral mucosa, and it compares these effects with those of smoking. Methodology: Oral mucosal samples were collected from 74 patients, consisting of 16 nonusers, 26 smokers, and 32 Maras powder users. Genes associated with oral cancer were selected and 90 microRNAs targeting these genes were identified. MicroRNA were isolated and purified using the microRNA isolation kit. MicroRNA were expressed using Fluidigm RT-PCR. Results: A positive correlation between the duration of Maras powder use with miR-31 expression levels, and a negative correlation between the Maras powder chewing time and miR-372 expression levels was found. In addition, there is a negative correlation between the amount of Maras powder consumed and expression levels of miR-375, miR-378a, miR-145, and miR-10b; moreover, another negative correlation is observed between the number of cigarettes consumed and the expression levels of miR-23a, miR-23b, miR-203a, miR-200b, and miR-375. However, miR-200b and miR-92a levels were downregulated significantly more in Maras powder users when compared with smokers and nonusers $(p<0.05)$. Conclusion: The results show both chewing Maras powder and smoking have an effect on deregulation of miR-200b and miR-92a expressions. This leads to the belief that assessing the expression of these two miRNAs is a promising noninvasive method of analysis, especially in mutagen exposures. Finally, large-scale and high-throughput studies may help to identify an extensive miRNA expression profile associated with tobacco use and improve the understanding of oral malignancies.

Keywords: MicroRNAs. Smokeless tobacco. Smoking. Mouth mucosa. 


\section{Introduction}

MicroRNAs (miRNAs) are endogenous small noncoding RNAs that function in messenger RNA (mRNA) silencing and in the post-transcriptional regulation of gene expression ${ }^{1}$. MiRNAs are important in various cellular processes, such as proliferation, differentiation, cell growth, and cell death ${ }^{2}$. Recent studies have discovered deregulated expression of miRNAs in oral infections, periodontal diseases, and oral cancer ${ }^{1,3,4}$. Xie, et al. ${ }^{1}$ (2011) performed a preliminary comparison of healthy and inflamed gingiva (10 healthy and 10 inflamed gingiva). They found 12 miRNAs, functioning in inflammatory processes and expressed differently, and reported a probable close relationship between miRNAs and periodontal diseases ${ }^{1}$. Similarly, Nahid, et al. ${ }^{5}$ (2011) found a persistent association between periodontal pathogens and miR-146a expression, suggesting miRNAs may promote periodontal diseases ${ }^{5}$. Likewise, understanding the relationship between miRNAs and oral malignancies has become increasingly important. Park, et al. ${ }^{6}$ (2009) evaluated and compared the miRNA expression profiles of oral squamous cell carcinoma patients and healthy controls, and this group identified two differentially expressed miRNAs (miR-200a and miR-125a) in carcinoma patients. In addition to the oral cancer, oral precancerous lesions also affect deregulation of miRNA expression ${ }^{4}$.

Tobacco products vary in the way they are consumed, for example, as cigarettes, cigars and cigarillos, which are smoked; also as smokeless tobaccos, such as chewing tobacco, snuffs, and dissolvable products. Yet, tobacco is mainly consumed in the form of manufactured cigarettes. According to the Global Adult Tobacco Survey conducted in 2015, smoking prevalence was $27.1 \%$ where smokeless tobacco use was not included 6 . However, Akbay and Kafas $^{7}$ (2017) reported that $16.7 \%$ of all participants were chewing MP, while $36.8 \%$ of all tobacco usage in South-East Turkey is of smokeless tobacco. The smokeless tobacco used in Turkey comes from Nicotiana rustica Linn and is called Maras powder (MP). To prepare for consumption, it is mixed with the ashes of wood - especially oak, walnut, or grapevine - , in approximate ratios of $1: 2$ or $1: 3$. The ash helps the absorption via oral mucosa by creating an alkaline environment ${ }^{8}$. The mixture is placed between the lips or the cheeks and gums/teeth and kept in the mouth for 5-10 minutes.

Despite the hazardous effects of tobacco on health, molecular alterations in the oral mucosa of tobacco chewers and smokers have not been fully investigated ${ }^{9}$. Experimental methods revealed miRNA expression in oral fibroblasts and oral keratinocytes is deregulated by the exposure to tobacco condensate ${ }^{9}$.

Because of this knowledge, this study hypothesized that smokeless tobacco use and smoking can deregulate miRNA expression profiles. Therefore, it aimed to analyze the expression profiles of 90 miRNAs (including miR-92a, miR-200b, miR-31, miR-372, miR-375, miR-378a, miR-145 and miR-10b, miR-23a, miR-23b, miR-203a, and miR-375) in a cross-sectional study to understand how chronic exposure of oral mucosa to tobacco affects the miRNA expression.

\section{Methodology}

This study was conducted at the Oral and Maxillofacial Surgery Department of the Faculty of Dentistry at Gaziantep University. Seventy-four male participants (aged between 21 and 54 years), consisting of 16 nonusers (no history of tobacco use), 26 smokers and 32 MP users, were included in this study. It was confirmed that smokers had never used MP and that MP users had never smoked.

This study followed the medical protocol set out by the Declaration of Helsinki and received ethical approval from Gaziantep University, Clinical Research Ethics Committee (2014/431). All participants signed a consent form stating their agreement to participate in the study. Data pertaining to these individuals were collected using a standardized questionnaire, which investigated age, sex, and smoking/chewing habits.

\section{Inclusion and exclusion criteria}

Volunteers between 18 and 65 years of age with no history of disease and acute/chronic inflammation were selected. Those who had been receiving a treatment regimen for less than 6 months were excluded. Those with presence or history of potentially premalignant oral lesions or oral cancer, dermatological diseases, oral infections, fractured jaw or previous surgery that could affect oral health, and those that consumed alcohol, narcotics, or tobacco products other than MP or cigarettes were also excluded. 


\section{Sample collection}

Oral samples were collected by scraping oral mucosa in one direction using a brush or surgical blade. The samples were stored at $-80^{\circ} \mathrm{C}$ in empty specimen containers until being anayzed ${ }^{1}$. Samples were collected from the buccal mucosa for smokers and nonusers, and from the application site for MP users.

\section{RNA isolation and cDNA synthesis}

Genes associated with oral cancer were selected based on the literature, and 90 miRNAs targeting these genes were identified using the TargetScan and miRDB databases. Furthermore, RNU6, Hs_SNORD68_11 and miRTC control, known to have relatively stable expression levels across different cell and tissue types $^{10}$, were used as an internal reference for normalization.

MiRNAs were isolated using the miScript primer assay kit (Qiagen, Santa Clarita, CA, USA), according to the manufacturer's instructions. Isolated miRNAs were reverse transcribed to generate complementary DNAs (cDNAs) with the miScript II RT kit (Qiagen, Santa Clarita, CA, USA). To increase the amount of cDNA, a pre-amplification step was performed (miScript PreAMP PCR kit - Qiagen, Santa Clarita, CA, USA). CDNA obtained after reverse transcription was stored at $-20^{\circ} \mathrm{C}$ until its use.

Real-time PCR was performed using a Rotor Gene 6000 Real-Time PCR Machine with the miScript SYBR Green PCR Kit (Qiagen, Santa Clarita, CA, USA) for miRNA expression.

\section{Bioinformatics and statistical analysis}

Threshold cycle $(\mathrm{Ct})$ values were obtained for the miRNAs and normalized using the internal control genes, respectively. All Ct analyses were performed using R Programming Language v3.2.2. Four miRNAs (hsa-miR-762, hsa-miR-371-5p, hsa-miR-4685-5p and hsa-miR-181d-3p) were excluded from the analyses for not having adequate $\mathrm{Ct}$ values. The expression profiles of 86 miRNAs were then analyzed. Due to the exponential nature of PCR data, data was transformed to log base 2 to achieve a normal distribution. The relative differences in expression (miRNA expression about the internal controls) were determined using the comparative threshold cycle (delta-delta $\mathrm{Ct}$ ) method. Fold change calculations were based on the formula $2^{-\triangle \Delta C T}$. The mean values of the control genes (RNU6,
Hs_SNORD68_11, and miRTC control) were obtained to calculate $2^{-\triangle \Delta C T}$.

Statistical analysis was performed using SPSS Version 22.0 (IBM, NY, USA). Variables were described using standard deviation (SD), standard error (SE), the range (minimum-maximum), and a $95 \%$ confidence interval for the mean. The One-way Anova test (F) was used to examine the differences between the miRNA expression profiles of the three groups. It was then followed by Duncan's post-hoc test for binary comparisons. The duration of usage, the amounts of MP or numbers of cigarettes consumed, and the amount of time that MP is chewed per day and the area where it is applied were analyzed using the Pearson's correlation test. $\mathrm{p}<0.05$ was considered statistically significant.

\section{Results}

All individuals who participated in this study were men. The mean age of the individuals was 32.02 $( \pm 8.13)$ and all study groups were age-homogeneous $(f=1.371, p>0.05)$. The intention was to investigate the expression profiles of 90 miRNAs; however, four miRNAs (miR-762, miR-371-5p, miR-4685-5p, and miR-181d-3p) were excluded from the study for not having adequate $\mathrm{Ct}$ values. The expression profiles of the 86 remaining miRNAs were analyzed (Figure 1 ).

Of the 86 miRNAs, only miR-92a and miR-200b showed different expressions between MP users, smokers, and nonusers. The mean ( $(\mathrm{SD})$ expression level of miR-92a was $5.979( \pm 1.22)$ in smokers, $5.343( \pm 1.62)$ in MP users, and $6.461( \pm 1.22)$ in nonusers (Figure 2), and the difference was statistically significant between the groups ( $f=4.790$, $\mathrm{p}=0.011$ ). Post-hoc analysis identified no significant difference between smokers and nonusers for miR$92 a$ expression $(p>0.05)$, while that of MP users was significantly different from that of smokers and nonusers $(p<0.05)$ (Table 1$)$

The mean $( \pm S D)$ expression level of miR-200b was $2.998( \pm 0.95)$ in smokers, $2.344( \pm 0.95)$ in MP users, and $3.127( \pm 1.04)$ in nonusers (Figure 3$)$. The difference in miR-200b expression was statistically significant between the groups $(f=3.606, p=0.032)$. Post-hoc analysis identified miR-200b significantly altered in MP users compared with nonusers $(p<0.05)$.

The mean duration of MP use was $9.68( \pm 5.59)$ 


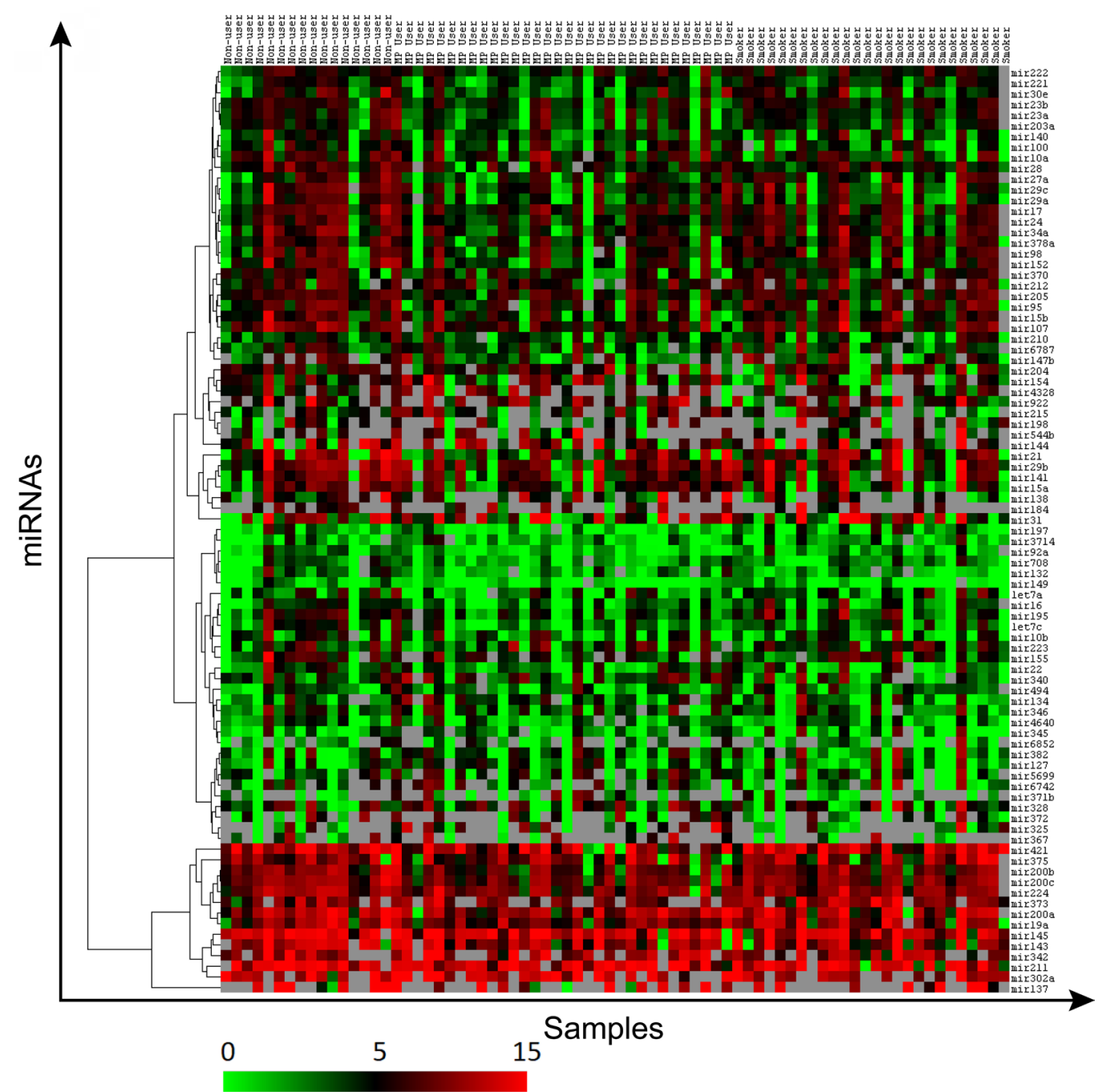

Figure 1-A heat map representing microRNA expression in nonusers, MP users and smokers. The clustering tree showing the concordance between miRNAs shown in the $\mathrm{Y}$ axis. Red tones on the heat map indicate higher expression levels than the median, while green tones show lower expression levels than the median

years, while the mean consumption amount was 0.64 $( \pm 0.46)$ packets per day. In addition, MP is chewed for 81.40 ( \pm 43.79$)$ minutes per day. The mean duration of smoking was $13.34( \pm 8.02)$ years, while the mean consumption amount was $1.00( \pm 0.32)$ packet per day.

It was determined that the duration of MP use was positively correlated with miR-31 expression $(r=0.444, p=0.016)$ and the MP chewing time per day was negatively correlated with miR-372 $(r=-0.628$, $p=0.005$ ).

While the consumption amount of MP was negatively correlated with miR-375 ( $r=-0.354$, $p=0.047)$, miR-378a $(r=-0.390, p=0.03), m i R-145$ $(r=-0.356, p=0.046)$ and miR-10b $(r=-0.357$, $p=0.045)$ expression, it was positively correlated with miR-138 expression ( $r=0.484, p=0.03)$.

The amount of cigarettes consumed was negatively correlated with miR-23a $(r=-0.428, p=0.033)$, miR23b $(r=-0.441, p=0.027)$, miR-203a $(r=-0.522$,

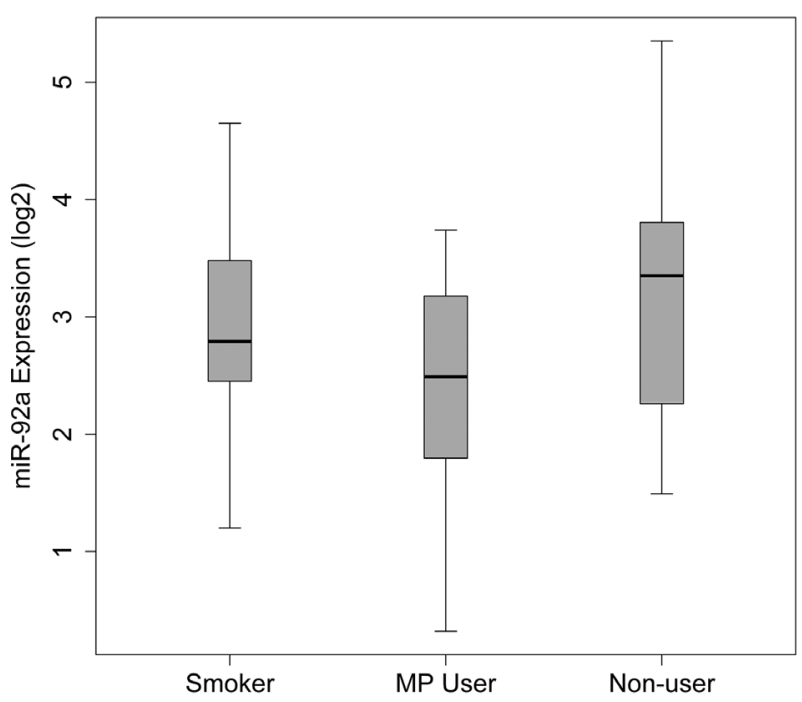

Figure 2- Boxplot of One-way Anova results for miR-92a expression

$p=0.008)$, miR-200b $(r=-0.410, p=0.042)$, and miR375 expression $(r=-0.475, p=0.016)$. 
Table 1- Differentially expressed miRNAs related to duration and consumption of MP and smoking

\begin{tabular}{ccccccc}
\hline miRNA & & N & Mean & SD & F & P \\
\hline \multirow{2}{*}{ miR-200b } & Smoker & $25^{*}$ & 2.998 & 0.958 & & 3.606 \\
& MP & 32 & 2.344 & 0.950 & & 0.032 \\
& Nonuser & 16 & 3.127 & 1.042 & & \\
miR-92a & Smoker & $25^{*}$ & 5.979 & 1.229 & & 0.011 \\
& MP & 32 & 5.343 & 1.620 & 4.790 & 1.229 \\
\hline
\end{tabular}

${ }^{*}$ Results of one sample were missing for related miRNAs

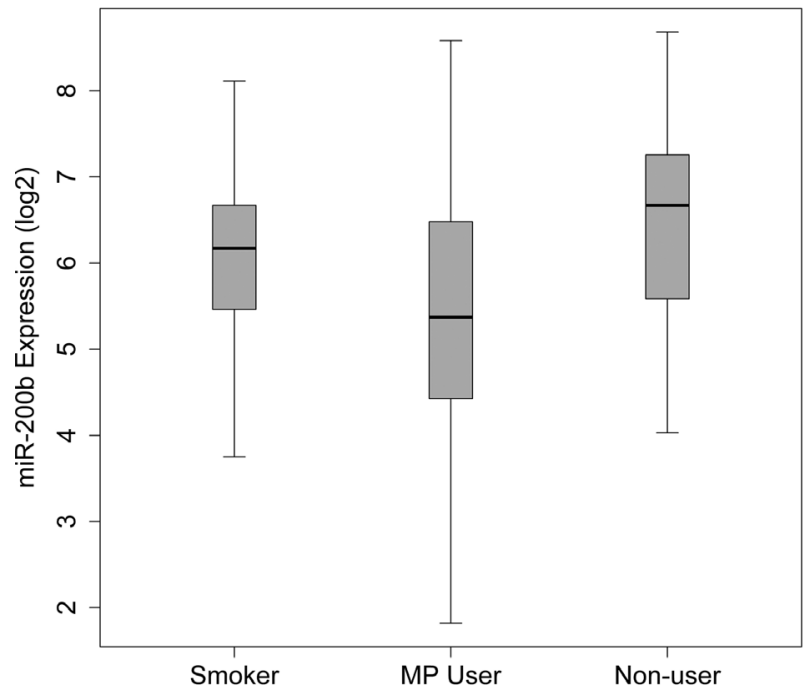

Figure 3- Boxplot of One-way Anova results for miR-200b expression

\section{Discussion}

Tobacco use (via smoking cigarettes or other forms of exposure to tobacco constituents) is closely associated with cardiovascular and respiratory diseases. It is also the most important risk factor for cancer and is responsible for the increased risk of death from all causes ${ }^{11}$. Besides, tobacco use affects inflammatory processes and causes dysplastic changes in oral tissues by changing the apoptotic function. A dysfunctional apoptotic system can contribute to the pathogenesis of many diseases, including oral pathologies ${ }^{10}$. MP causes genotoxic changes in oral mucosa ${ }^{10,12}$. An epidemiological study revealed that $9 \%$ of 80 individuals using MP had dysplasia, carcinoma in situ, and cancer correlated with the duration of consumption ${ }^{13}$.

MP consumption is rare among women in Turkey. Therefore, only male subjects were included in our study. Concerning this topic, Akbay and Kafas ${ }^{8}$ (2017) reported that among participants using MP, $1.4 \%$ were women and $25.1 \%$ were men.

Tobacco products modulate the turnover of epithelial and mesenchymal tissues ${ }^{14}$ and inhibit cell apoptosis, playing a significant role in oral carcinogenesis ${ }^{15}$. Bhat, et al. ${ }^{10}$ (2018) reported that tobacco use alters miRNA expression in oral cells. This leads us to investigate the miRNA expression of oral mucosa in two frequently used forms of tobacco. To the best of our knowledge, we are the first group to investigate the effect of MP use on miRNA expression. From the 90 miRNAs analyzed, only miR-92a and miR-200b showed different expressions between MP users, smokers, and nonusers. The downregulation of the miR-200 family is allegedly a sign of inhibitory effects on cell proliferation, invasion, and metastasis and functions as a tumor suppressor ${ }^{16}$. In our study, the mean expression level of miR-200b was lower in MP users than in smokers and in nonusers, and a significant difference was observed between MP users and nonusers. Similar to our findings, Bhat, et al. $^{10}$ (2018) determined that miR-200b found to be downregulated in oral keratinocytes exposed to cigarette smoke. MiR-92a is suggested to serve as an oncogene or tumor suppressor in different cancers. In this study, the mean expression level of miR-92a was lower in MP users than in smokers and in nonusers, and there was a significant difference between MP users and smokers, and between MP users and nonusers.

Our results showed miR-31 is positively correlated with MP use, similar to those of Bhat, et al. ${ }^{10}$ (2018), who found upregulation of miR-31 in smokeless tobacco-treated oral cells. MiR-31 is also upregulated in oral premalignant epithelium and epithelial dysplasia and in several neoplasms, including head and neck cancers $^{17}$.

The number of cigarettes consumed was negatively correlated with miR-23a, miR-23b, miR-203a, miR200b, and miR-375 expression. Many studies have been conducted to evaluate the effect of smoking on miRNA regulation. Smoking has been reported to reduce miR-203a expression in rectal cancer ${ }^{18}$. Also, $\mathrm{Ma}$, et al. ${ }^{19}$ (2014) stated that the dysregulation 
of miR-23a and miR-23b may be implicated in the progression of human gastric cancer, and the combined expression of miR-23a and miR-23b seems to be a valuable marker for prognosis of this disease ${ }^{19}$.

This study, interestingly, determined that both the amount of MP and number of cigarettes consumed were negatively correlated with miR-375. Similarly, Conickx, et al. ${ }^{14}$ (2017) showed the expression of miR375 decreased significantly in the lungs of mice after exposure to cigarette smoke for 24 weeks ${ }^{13}$.

MiRNA profiling was performed on oral mucosa, although blood could be tested to determine the correlation between tissue and blood. In addition, histological analysis could be conducted to support our findings. The sample size is our limitation in this study, as MP is usually consumed with cigarettes and/ or is used as an alternative to smoking. Multicenter longitudinal studies investigating larger sample sizes are needed to generalize our findings. In future studies, examining the changes in existing miRNA expressions after the cessation of tobacco use will also be necessary.

\section{Conclusions}

The results of our study show the use of MP and smoking deregulate miRNA expression (miR-200b and miR-92a). This leads to the belief that assessing miRNA expression is a promising noninvasive method of analysis, especially in the case of mutagen exposures. Finally, large-scale and high-throughput studies may help to identify extensive miRNA expression profiles associated with tobacco use and improve the understanding of oral malignancies.

\section{Ethical approval}

This study was approved by Gaziantep University Clinical Research Ethics Committee. All procedures involving human participants were conducted according to the ethical standards of the institutional and/or national research committee and with the 1964 Declaration of Helsinki and its later amendments or comparable ethical standards.

\section{Acknowledgement}

The authors would like to thank Prof. Dr. Metin Gungormus for checking the content of this article and DNAFect Inc for assisting in bioinformatics analysis.

\section{Funding}

This work was supported by the Scientific and Technical Research Council of Turkey (Tübitak) under Grant No. 115 S018.

\section{Author contributions}

Taş, Betül: Conceptualization (Lead); Data curation (Equal); Formal analysis (Equal); Funding acquisition (Lead); Investigation (Lead); Methodology (Lead); Project administration (Lead); Resources (Equal); Software (Equal); Supervision (Equal); Validation (Equal); Visualization (Equal); Writing-original draft (Equal); Writing-review \& editing (Equal). Güre, Ali Osmay: Data curation (Equal); Formal analysis (Equal); Supervision (Equal); Visualization (Equal).

\section{References}

1- Xie YF, Shu R, Jiang SY, Liu DL, Zhang XL. Comparison of microRNA profiles of human periodontal diseased and healthy gingival tissues. Int J Oral Sci. 2011;3(3):125-34.

2- Manasa VG, Kannan S. Impact of microRNA dynamics on cancer hallmarks: an oral cancer scenario. Tumor Biol. 2017;39(3).

3- D'aiuto F, Suvan J. Obesity, inflammation, and oral infections: Are microRNAs the missing link? J Dent Res. 2012;91(1):5-7.

4- Roy R, Singh R, Chattopadhyay E, Ray A, Sarkar N De, Aich R, et al. MicroRNA and target gene expression based clustering of oral cancer precancer and normal tissues. Gene. 2016;15(593(1)):58-63.

5- Nahid MA, Satoh M, Chan EKL. MicroRNA in TLR signaling and endotoxin tolerance. Cell Mol Immunol. 2011;8(5):388-403.

6- Asma S, Mackay J, Yang Song S, Zhao L, Morton J, et al. The GATS Atlas [Internet]. 2015 [cited 2019 Oct 17]. p. 38-9. Available from: http://gatsatlas.org/pdf/mobile/index.html\#p=38

7- Akbay C, Kafas A. Analysis of factors affecting cigarette smoking and Maras powder use among adults in the urban area of Kahramanmaras. KSU J Nat Sci [Internet]. 2017;20(3):276-82. Available from: https:// dergipark.org.tr/tr/download/article-file/272908

8- Dagli AF, Sahin N, Bozdag Z, Ucer O, Akatli AN, Artas G, et al. Cytological and cytomorphometric characteristics of buccal mucosa cells from smokeless tobacco users. Diagn Cytopathol. 2017;45((11)):97682.

9- Bhat MY, Advani J, Rajagopalan P, Patel K, Nanjappa V, Solanki HS, et al. Cigarette smoke and chewing tobacco alter expression of different sets of miRNAs in oral keratinocytes. Sci Rep. 2018;4(8(1)):7040. 10- Solanki S, Advani J, Kumar M, Kumar P, Bhagat $\mathrm{H}$ et al. miRNA profiling of bladder cells chronically exposed to cigarette smoke condensate and its vapor. Res Reports. 2017;1:e1-15.

11- World Health Organization. Chapter 1: Burden: mortality, morbidity and risk factors. Glob Status Rep Non-communicable Dis 2010. 2011;9-31.

12- Conickx G, Avila Cobos F, Van Den Berge M, Faiz A, Timens W, Hiemstra PS, et al. MicroRNA profiling in lung tissue and bronchoalveolar lavage of cigarette smoke-exposed mice and in COPD patients: A translational approach. Sci Rep. 2017;9(7(1)):12871.

13- Erenmemişoğlu A, Üstün H, Kartal M. Carcinoma of buccal mucosa in smokeless tobacco users: a preliminary study of the use of cytology for early detection. Cytopathology. 1995;6(6):403-8. 
14- Misra A, Rai S, Misra D. Functional role of apoptosis in oral diseases: An update. J Oral Maxillofac Pathol. 2016;20(3):491-6.

15- Wang C, Niu W, Chen H, Shi N, He D, Zhang M, et al. Nicotine suppresses apoptosis by regulating a7nAChR/Prx1 axis in oral precancerous lesions. Oncotarget. 2017;24(8(43)):75065-75.

16- Varol N, Konac E, Gurocak OS, Sozen S. The realm of microRNAs in cancers. Mol Biol Rep. 2011;38(2):1079-89.

17- Schlosser K, McIntyre LA, White RJ, Stewart DJ. Customized internal reference controls for improved assessment of circulating micrornas in disease. PLoS One. 2015;26(10(5)):e0127443.
18- Mullany LE, Herrick JS, Wolff RK, Stevens JR, Slattery ML. Association of cigarette smoking and microRNA expression in rectal cancer: insight into tumor phenotype. Cancer Epidemiol. 2016;45:98107.

19- Ma G, Dai W, Sang A, Yang X, Gao C. Upregulation of microRNA$23 a / b$ promotes tumor progression and confers poor prognosis in patients with gastric cancer. Int J Clin Exp Pathol. 2014;1;7(12):883340. 